PTH-100 GASTWELL: A NOVEL, INTEGRATED HEALTH PSYCHOLOGY SERVICE FOR INDIVIDUALS WITH INFLAMMATORY BOWEL DISEASE

${ }^{1}$ Jacqueline Lavallée*, ${ }^{2}$ Karen Kemp, ${ }^{2}$ Sophia Joseph, ${ }^{2}$ Scott Levison, ${ }^{3}$ Chris Bundy. ${ }^{1}$ University Of Manchester, Manchester, UK; ${ }^{2}$ Manchester University NHS Foundation Trust, Manchester, UK; ${ }^{3}$ Cardiff University, Cardiff, UK

\subsection{6/gutjnl-2019-BSGAbstracts. 159}

Background Inflammatory Bowel Disease (IBD) presents challenges to psychological coping and adjustment, impacting health outcomes and disease activity. In the UK individuals with IBD have limited access to health psychology support services which can improve the quality of life and management of long-term conditions. We developed and evaluated an evidence led, integrated health psychology clinic (GastWell) within the IBD service in a large teaching hospital.

Methods Anxiety, depression and illness perception data were collected from 126 patients attending an outpatient IBD nurse clinic to gain a greater understanding of the overall need for a psychological service and to assess whether the GastWell clinic is capturing all those in need of health psychology support. Specialist IBD Health Psychologists delivered brief, behaviour change focused interventions within the GastWell clinic. Following referrals from the IBD team, patients were assessed and offered assistance in adjusting to and managing their IBD, including reducing smoking and alcohol consumption. We evaluated the GastWell service using validated patient-reported outcome measures before and after intervention. Measures include the Short IBD questionnaire, Brief Illness Perceptions questionnaire and Hospital Anxiety and Depression Scale (HADs).

Results 31 patients were referred to the monthly GastWell clinic between October 2016 and October 2018. The most common reason for referral was for support in adjusting to and managing IBD. Compared to the IBD population attending the outpatient IBD nurse clinic, those referred to the GastWell clinic had a more threatening view of their IBD and higher anxiety and depression scores. Patient reported outcomes suggest reductions in illness perceptions, anxiety and depression scores and an increase in health-related quality of life from pre to post intervention.

Conclusion Our service evaluation suggests that those requiring health psychology support are being correctly referred into the GastWell clinic, and that the interventions are appropriate and effective in assisting patients with adjusting to and managing their IBD. In addition, we have demonstrated reductions in illness perceptions, anxiety and depression scores that are lower than the general IBD population at this site. Greater access to integrated health psychology IBD services is necessary and may benefit all individuals with IBD.

*Higher score indicates higher quality of life.

**Higher scores indicate a more threatening view of the illness.

$* * * 0-7=$ normal; $8-10=$ borderline abnormal; $11-21=$ abnormal.

\section{PTH-101 INFLIXIMAB THERAPEUTIC DRUG MONITORING: REACTIVE VS PROACTIVE APPROACH - UNIVERSITY HOSPITALS OF LEICESTER (UHL) EXPERIENCE}

Hui Lee*, Patricia Roberts, Sanjeev Pattni. University Hospitals Leicester, Leicester, UK

\subsection{6/gutjnl-2019-BSGAbstracts. 160}

Introduction and aims Infliximab (IFX) is a recognised effective therapy in moderate to severe inflammatory bowel disease (IBD). There has been an evolvement in IBD management towards a treat-to-target approach, with therapeutic drug monitoring (TDM) playing an increasingly essential part to optimise personalised care. Presently there is no standardised guidance defining a treatment strategy for patients on IFX although several treatment algorithms have been proposed. The aim of this project is to evaluate the utility of IFX TDM in patients with IBD, in our hospital.

Methods This is a single centre retrospective cohort analysis of patients with IBD receiving IFX that had TDM between January 1st2017 to June 30th2018. The indications of requests, IFX trough levels, presence of anti-drug antibody (ADA) and clinical outcomes were analysed. The outcome was to compare the clinical outcomes for TDM done reactively versus proactive monitoring. Reactive TDM was requested in a patient due to active symptoms or asymptomatic patients with evidence of active inflammation on radiology and/or endoscopy.

Results A total of 54 patients were included in this study. 37 had Crohn's Disease (CD) and 17 had Ulcerative Colitis (UC) with a mean age of 43 years and 51 years respectively. These patients were tested at least once within 71 TDM results obtained. Out of these TDM results, 49\% (n=35) were requested reactively, 39\% $(\mathrm{n}=28)$ for proactive monitoring and $12 \%(n=8)$ unclear reasons. The mean and median infliximab trough levels were $3.8 \mu \mathrm{g} / \mathrm{ml}$ and $4.7 \mu \mathrm{g} / \mathrm{ml}$ respectively (range $<0.4$ to greater than $10 \mu \mathrm{g} / \mathrm{ml}) .37 \%(\mathrm{n}=20)$ had subtherapeutic levels with positive ADA.

Results of TDM in 60\% $(n=21)$ and $75 \%(n=21)$ in the reactive and proactive group respectively did not alter patients' management $(p=0.284) .17 \% \quad(n=6)$ switched to an alternate biologic agent in the reactive group and 7.1\% $(n=2)$ in the proactive made a switch. There was a need of $5.7 \%$ $(n=2)$ and $7.1 \%(n=2)$ in the reactive and proactive groups respectively for intestinal surgery. $8.5 \%(n=3)$ stopped biologic therapy in the reactive group and nil in proactive group.

Conclusion In our experience, IFX TDM reactively or proactively, did not alter clinical outcome/management in the majority or our patients. It is likely that TDM served to ensure on going continuation of the drug. We were able to change patient's treatment (biologic switch) in a small number of patients through proactive monitoring and stop therapy in a small proportion of patients when taking a reactive TDM approach. Ideally TDM pathways need to be formulated to deliver a more organised personalised care with anti-TNF prescribing. 